\title{
Chitin-gold nanocomposite film and electro-optical properties
}

\author{
Zameer Shervani* \\ Nanomaterials Production Division, Food and Energy Security Research and Product Center, Aoba-ku, Sendai 980-0871, Japan
}

\begin{abstract}
Chitin, the second most abundant biomolecule on earth after cellulose, was used to prepare 25-40 nm diameter chitin nanofibers (CNFs) in wet acidic condition from crab shells. Using CNFs suspension, neat CNFs and gold nanoparticles (Au NPs) embedded $70 \mu \mathrm{m}$ thin printable films were prepared. Electrical and optical properties of these biodegradable organic-inorganic hybrid nanocomposite of CNFs-Au NPs have been reported first. Filling the network of tricyclodecane dimethylol dimethacrylate (TCDDMA) resin with CNFs-Au NPs blend and subsequent polymerization using photoinitiator 2-hydroxy-2-methylpropiophenone gave a transparent thin film. CNFs-Au NPs film had no transmittance when impregnated with (TCDDMA) resin, however, polymerised film became 64\% transmitted to normal visible light. Conductance of base CNFs material was enhanced by blending with Au NPs as measured by high sensitivity impedance analyser. Au NPs size and diameter of CNFs were determined by recording field emission scanning electron micrograph (FE-SEM). X-ray diffraction of the film confirmed the bands of crystalline chitin and nanometallic gold. This new environmentally benign composite has been recognized as a strong candidate for high performance substrate for solar cell, electrical, electronic, and optical devices flexible display, electronic paper, and optical waveguide.
\end{abstract}

\section{Introduction}

Biomaterials are playing important and increasing roles in production of organic and inorganic nanocomposites as they are cheap, non-toxic, water soluble and easy to remove when nanomaterials are taken on substrates. Carbohydrates monosaccharide $\beta$-D-glucose and polysaccharide soluble starch were used as stabilizing agents to prepare gold and silver NPs. Homogeneous gold NPs of $5.3 \mathrm{~nm}(\sigma=0.7)$ diameter were obtained when gold salt in glucose solution was reduced by $\mathrm{NaBH}_{4}$ $[1,2]$. Similarly silver NPs of $15 \mathrm{~nm}$ were prepared by reduction of silver salt in soluble starch by reducing agent $\beta$-D-glucose [3]. Another biomaterial, chitin, a polysaccharide carbohydrate polymer is a natural biocompatible, biodegradable, bioadsorbable, and non-toxic material obtained from a number of sources including crab or prawn shells. Chitin nanofibers (CNFs) are prepared from dry crab or prawn shell chitin powder by fibrillation process [4,5]. If not used, chitin along with other fish industry wastes are thrown as industrial waste. There is much demand and scope of chitin and its derivatives [6-8] in pharmaceuticals and cosmetics industries if chitin fibrillated to CNFs.

Among biodegradable polymers chitin is most abundant carbohydrate, second to cellulose, in nature with annual production $10^{11}$ tons per year [9]. CNFs because of nanometer size width have been used as reinforcement agents in a number of resins to enhance mechanical and thermal properties of composites [10]. Chitin whiskers of $500 \mathrm{~nm}$ length and $50 \mathrm{~nm}$ diameter were used as filler to reinforce soy protein isolate (SPI) plastic [11]. Tensile strength and Young's modulus of composite sheet increased compared to neat SPI composite. Weatherability of SPI-chitin composite was also increased considerably. Authors prepared CNFs film and CNFs-natural rubber (NR) porous sheets [12]. CNFs film was prepared by mixing chitin water dispersion with 1-allyl-3-methylimidazolium bromide (AMIMBr) in a certain ratio, mixture was allowed to stand at room temperature for $24 \mathrm{~h}$ and subsequently heated for $24 \mathrm{~h}$ at $100^{\circ} \mathrm{C}$ thus chitin ion gel was obtained. CNFs ion gel was soaked and sonicated in methanol to obtained CNFs ions dispersion. Pure CNFs film was prepared by filtration of CNFs ions dispersion. In second step, above CNFs film was dissolved in aqueous ammonia and after several steps of processing and mixing with NR, CNFs-NR composite sheet was obtained. CNFs flexible transparent paper was prepared by dissolving chitin powder in hexafluoroisopropanol (HFIP), soluble chitin solution in HFIP was poured in polypropylene dish and kept in fume hood for $2 \mathrm{~d}$ to get a chitin-HFIP alcogel [13]. Alcogel was casted to a Chitin NF paper by centrifuging the polypropylene dish. Obtained CNFs paper was sandwiched dried between glass plates and hot vacuum pressed to make it straight for use.

In the present research, we have prepared first a transparent CNFs$\mathrm{Au}$ NPs composite $70 \mu \mathrm{m}$ thin film and study its electrical and optical properties. As described above transparent CNFs based composite films had been prepared by researchers but CNFs-nanometallic materials preparation has not been done and investigated so far. New organic-inorganic transparent composite will expand applications of CNFs in pharmaceutical, cosmetic, electrical, electronic, and solar cell industries.

\section{Materials and methods}

$\mathrm{NaBH}_{4}$ and gold precursor salt hydrogen tetrachloroaurate (III) trihydrate $\left(\mathrm{HAuCl}_{4} \cdot 3 \mathrm{H}_{2} \mathrm{O}\right)$ were obtained from Wako Pure Chemical

Correspondence to: Zameer Shervani, Director, Food and Energy Security Research and Product Center, Sendai, Japan, E-mail: shervani.nanotek@ gmail.com

Key words: Biodegradable composite, Gold nanopartilces, Chitin nanofibers, Hybrid nanocomposite

Received: September 20, 2017; Accepted: October 06, 2017; Published: October 09,2017 
Industries Ltd. Poly (methyl vinyl ether) PMVE, triblock copolymer used as stabilizing agent for Au NPs was from Aldrich. Commercial chitin powder of crab shells used for CNFs preparation was from NACALAI TESQUE, INC. Tricyclodecane dimethylol dimethacrylate (TCDDMA) was obtained from Chisso Corporation. Nonionic photoinitiator 2-hydroxy-2-methylpropiophenone was purchased from Tokyo Kasei Kogyo Co. Ltd.

\section{Preparation of neat CNFs film}

Mushi [14] has described detail method of preparation and characterization of CNFs film from crab shells. In brief, $1 \mathrm{wt} \%$ commercial chitin powder slurry was prepared in water and $\mathrm{pH}$ was adjusted by acetic acid to 3 . Slurry was stirred over night at room temperature [15]. By vacuum filtering wet chitin cake was obtained. Chitin cake was dissolved once more in water and $\mathrm{pH}$ was adjusted again to 3 by acetic acid. For fibrillation slurry was passed through a grinder model MKCA6-3; Masuko Sangyo Co., Ltd. operating at 1500 $\mathrm{rpm}$. After two cycles of grinding slurry was changed to highly viscous gel of CNFs. These CNFs were used for neat CNFs and CNFs-Au NPs composite film preparations.

\section{Preparation of pre-organized Au NPs}

A measured amount of Au salt stock solution $(0.05 \mathrm{M})$ was added to polymer PMVE (0.6 wt\%)-water dispersion to get a final Au salt concentration $10^{-3} \mathrm{M}$. Au salt was reduced in polymer dispersion by freshly prepared $\mathrm{NaBH}_{4}$ stock solution $(0.1 \mathrm{M})$ in ethanol to obtain Au NPs.

\section{Preparation of CNFs-Au NPs film}

$16 \mathrm{wt} \% \mathrm{CNF}$ dispersion were prepared in water then pre-organized $\mathrm{Au}$ NPs prepared in soluble polymer were mixed in diluted NFs suspension in equal proportion. The mixture was stirred and sonicated for 30 minutes each. Thin films of neat CNFs and CNFs-Au NPs composite were prepared by taking dispersion containing $20 \mathrm{~g}$ CNFs in a beaker. Preparations were vacuum stirred for $30 \mathrm{~min}$ to remove air bubble then filtered under vacuum on Teflon filter and washed three times with ethanol. Film was separated from Teflon filter and pressed dried at $100^{\circ} \mathrm{C}$ to remove moisture. Thus a $70 \mu \mathrm{m}$ thin dried film of CNFs-Au NPs was finally obtained. Mother liquor obtained during filtration was colorless.

\section{Results and discussion}

\section{FE-SEM of CNFs film from crab shell}

Figure 1 is the field emission scanning electron micrograph (FESEM) of CNFs film obtained by fibrillating slurry of chitin in acidic medium as described in section 2.2. The SEM image is of three grinding cycles of chitin slurry. We investigated the effect of grinding cycles vs the aspect ratio of CNFs obtained and phase stability of CNFs as a function of number of passes through grinder. It was noticed that two pass grinding suspension of diluted CNFs phase separated in $4 \mathrm{~h}$ while three pass suspension remained stable and did not phase separate even keeping for 6 months. Bar diagram (Figure 2) of aspect ratio vs number of grinding cycles showed that three grinding cycles preparation fibers had maximum aspect ratio. FE-SEM images compared of two, three, five, and ten grinding cycles showed fibers prepared by three cycles were elongated, thin, and well separated compared to others. Increasing number of grinding cycles more than three breaks fibers to smaller segments. Thus fibers prepared in three grinding cycles was best preparation of width $25-40 \mathrm{~nm}$ range.

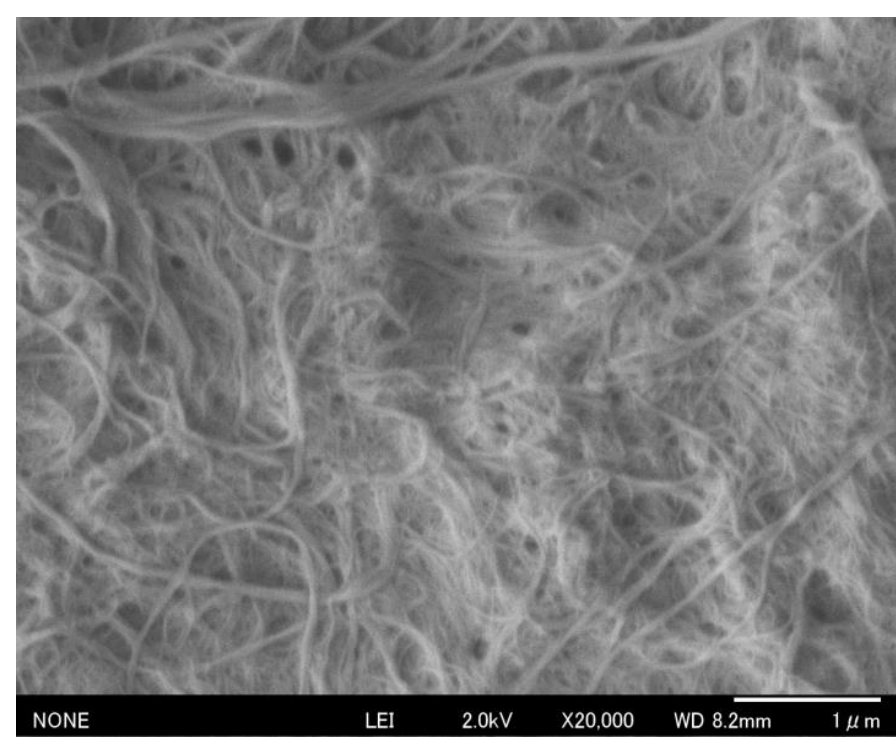

Figure 1. FE-SEM image of three cycles pass grinding of ; scale: $1000 \mathrm{~nm}$

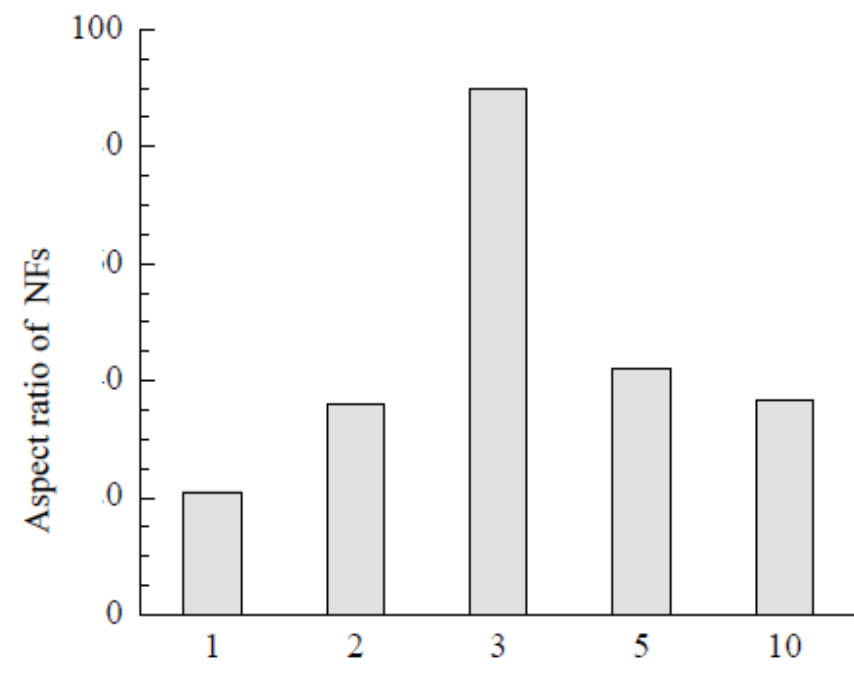

Grinding cycles

Figure 2. Grinding cycles vs aspect ratio

\section{Neat CNF and CNF-Au NPs composite films}

White thin film of neat CNFs (Figure 3a) and dark reddish film of CNFs-Au NPs (Figure 3c) were prepared as described in experimental section 2.4. Diluted CNFs suspension of chitin content $0.18 \mathrm{wt} \%$ was mixed with pre-organized Au NPs stabilized in PMVE polymer solution. A light chocolate color of CNFs-Au NPs suspension was phase separated on keeping mixture unstirred for $4 \mathrm{~h}$. Supernatant mother liquor is colorless indicative that all Au NPs mass transferred from polymer to more electronegative moieties of chitin molecules shedding the capping shell of polymer as the polymer PMVE is soluble in water. Chitin to gold metal content in composite film was 230:24 mg, respectively. X-ray diffraction pattern (Figure $4 \mathrm{a}, \mathrm{b}$ ) confirmed the chitin and Au metallic content in the composite films. Well known chitin bands with planes in bracket at two theta positions: $9.6^{\circ}(020)$, $19.7^{\circ}(110), 23.7^{\circ}(130)$ and metallic gold at $38.8^{\circ}(111), 44.3^{\circ}(200)$, $65.1^{\circ}(220), 77.1^{\circ}(311)$ were obtained. FE-SEM image (Figure 5) of the film showed Au NPs of size $19 \mathrm{~nm}(\sigma=7)$ were embedded in the film. 


\section{Optical property of neat CNF and CNF-Au NPs composite films}

Figure $3 \mathrm{~b}$ and $\mathrm{d}$ are TCDDMA resin soaked CNFs and CNFs- $\mathrm{Au}$ NPs composite films, respectively, cured by UV irradiation in a chamber (SPOT CURE SP-7, Ushio Inc.) for $10 \mathrm{~min}$ at $40 \mathrm{~mW} \mathrm{~cm}^{-1}$ in presence of radical polymerization initiator 2-hydroxy-2-methylpropiophenone. After soaking in resin and subsequent polymerization films became transparent as thumbs holding the film from behind are clearly visible. Weatherability and toughness of treated film also increased. A cutout strip of each film was prepared to fit in UV-vis spectrophotometer (JASCO-V550) holder to record \% transmittance of the resin treated and untreated films. As shown in Figure 6 of visible spectral recording,
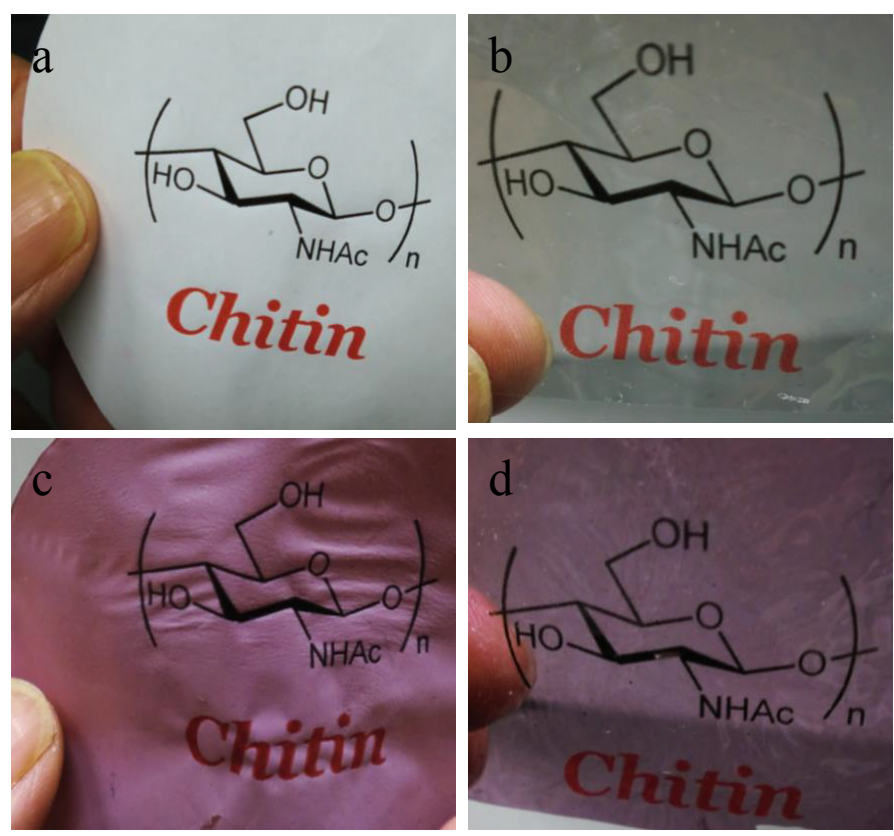

Figure 3a-d. Optical transparency of neat chitin (a) and Au NPs blended chitin (c) films without TCDDMA treatment and TCDDMA soaked neat chitin (b) and and Au NPs blended chitin (d); TCDDMA treated film was subsequent polymerized with photoinitiator 2-hydroxy-2- methylpropiophenone and cured under UV irradiation

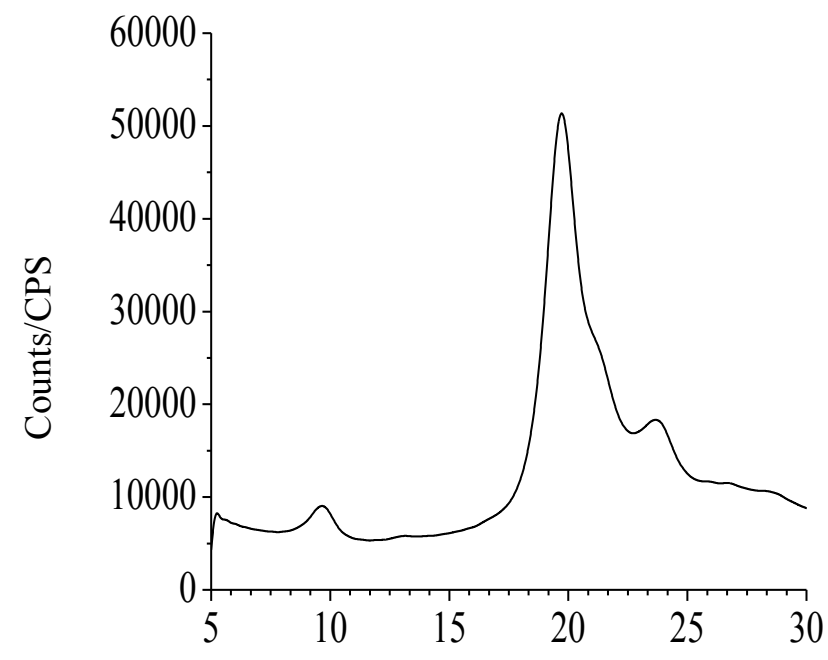

Two theta/degree

Figure 4a. XRD pattern of chitin part of CNF-Au NPs composite

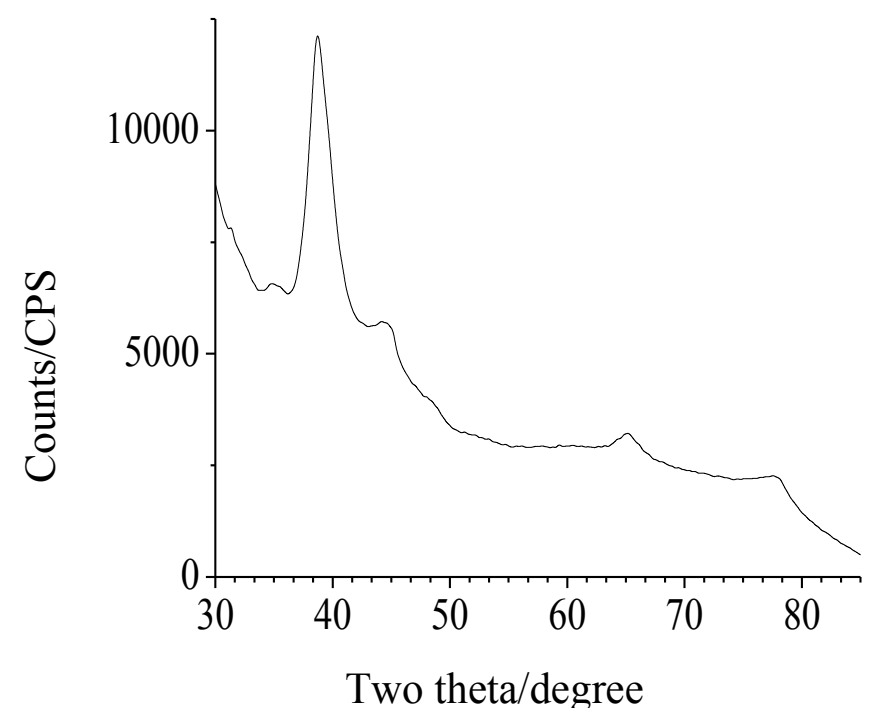

Figure 4b. XRD pattern of Au metallic part of CNF-Au NPs composite

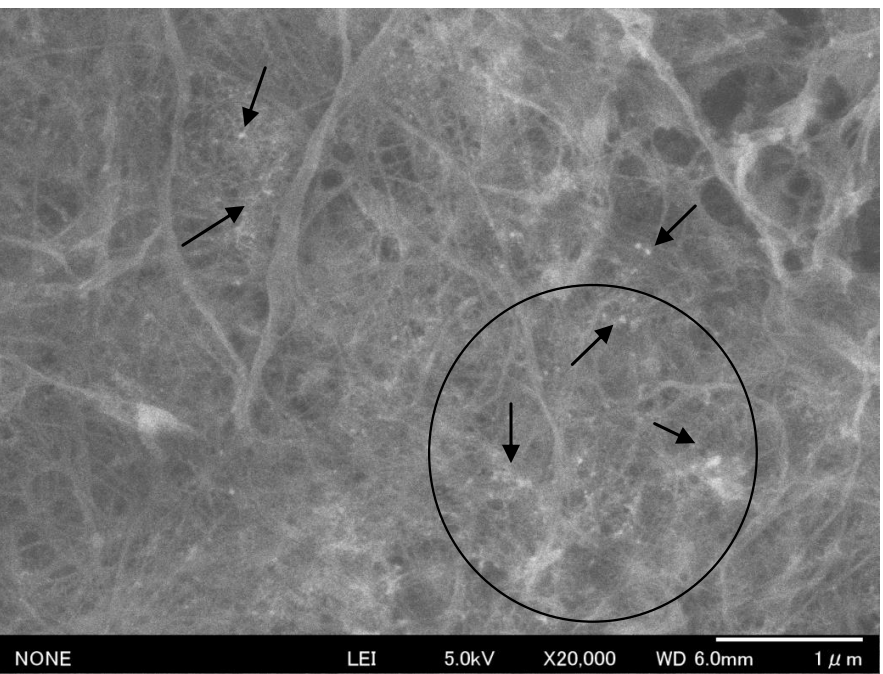

Figure 5. FE-SEM image of CNFs-Au NPs composite film showing Au NPs

untreated neat CNFs sheet and CNFs-Au NPs loaded sheet were not transparent at all. While neat TCDDMA resin treated and polymerized sheet were highly $(80 \%)$ transparent in visible region at $\lambda=750 \mathrm{~nm}$. However, Au NPs loaded became $62 \%$ transparent at $\lambda=750 \mathrm{~nm}$ when treated with resin and polymerized. At $550 \mathrm{~nm}$ a broad band of Au NPs was observed, the band is broad due to NPs binding with chitin polar moieties. There are two phenomenon making resin treated CNFs and CNFs-Au NPs composite film transparent. CNFs work as a nanofillers inside the resin network, due to nanosize of fibers, the material become transparent. Other important phenomena is refractive index (RI) of composite matrix. Matching of RI of composite and the interface is important to obtain a transparent material. RI of chitin is 1.54 and of air is 1.00 thus there exists void volume of air in sheet of different RI [12]. Thus neat CNF sheet or Au NPs blended untreated chitin sheet has no transmittance. When NFs sheet impregnated with resin TCDDMA of $\mathrm{RI}=1.51$, RI of neat chitin sheet and interface TCDDMA reached to near matching values thus impregnated neat CNF sheet became $80 \%$ transparent and Au NPs blended sheet had 62\% transmittance. Figure 3 showed that neat CNFs or CNFs-Au NPs sheets can be used similar to an ordinary paper as ink jet printing of two different patterns has been 
demonstrated. Printing on such transparent thin $(70 \mu \mathrm{m})$ composite sheet can be applied for wire printing needed in minute electronic devices and can be used as electronic paper.

\section{Electrical property of films}

Figure 7 is impedance plot of neat CNF and CNF-Au NPs sheets as measured by impedance analyzer of model HP $4192 \mathrm{~A}$, operating at two probes AC method. An electrode of $1.76 \mathrm{~cm}^{2}$ area of $70 \mu \mathrm{m}$ thin film was prepared by coating silver metal paste on both sides of film. Silver coated film was sandwiched between two copper metal sheets that acted as current collector. Semicircle of real and imaginary components of impedance of $\mathrm{Au}$ NPs blended $\mathrm{CNF}$ film was smaller than neat $\mathrm{CNF}$ film base material indicative that Au metallic NPs blending with chitin has enhanced the conductivity of chitin film $[16,17]$. Thus conductance of chitin sheet or similar biomolecules can be enhanced by doping nanometallic particles.

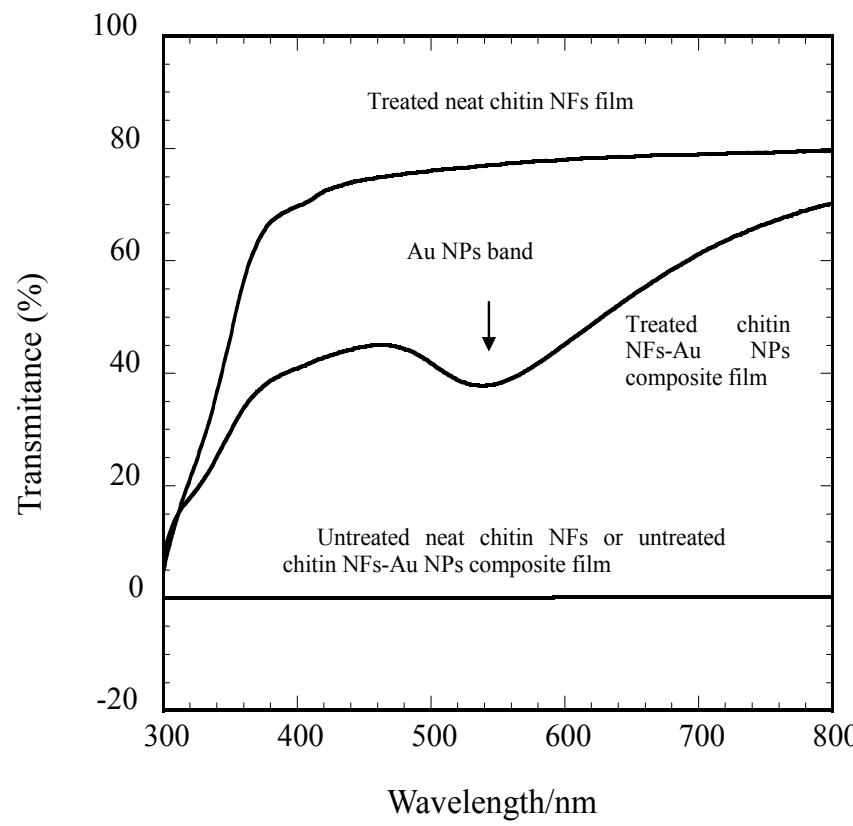

Figure 6. \% transmittance vs wavelength plots of untreated neat chitin NFs, untereated chitin NFs-Au NPs composite, treated neat NFs, and treated chitin NFs-Au NPs films; band at $\lambda=520$ is of Au NPs

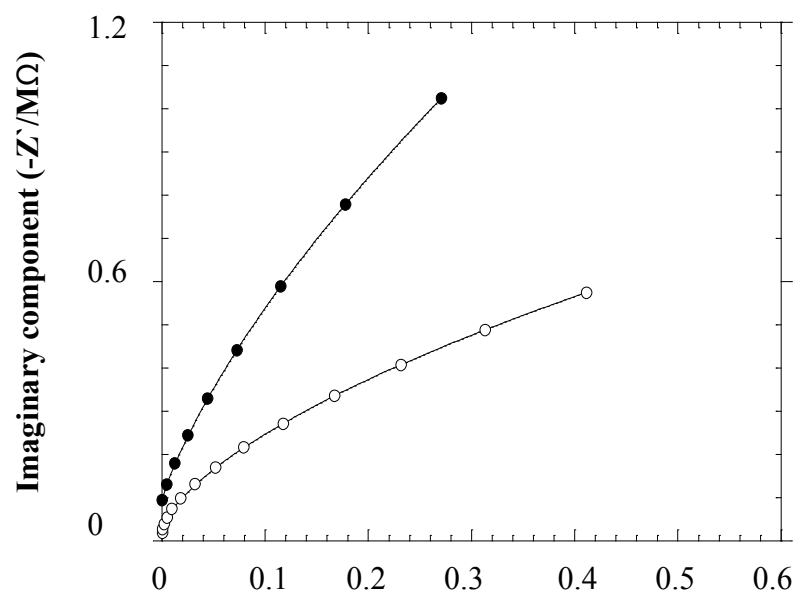

Real component $Z / M \Omega$

Figure 7. Impedance plot of neat chitin (•) and Au NPs blended chitin (o) nanofiber sheet

\section{Conclusion}

CNFs-Au NPs composite thin $(70 \mu \mathrm{m})$ film was successfully prepared by blending pre-organized Au NPs with diluted CNFs suspension. On addition of CNFs suspension to NPs-PMVE polymer solution, NPs were mass transferred onto the polar moieties of chitin molecules. Impregnating nontransparent CNFs and CNFs-Au NPs films with TCDDMA resin made films optically transparent. Blending of Au metallic NPs with CNFs film enhanced electrical conductance of base material chitin film.

\section{Conflict of interest statement}

There is no conflict of interest in this research academically or financially. Research is original and has been done independently for the research center's nanotechnology product manufacturing. The purpose of research is to use seafood industrial waste for high value applications and research.

\section{References}

1. Shervani Z, Yamamoto Y (2011) Carbohydrate directed synthesis of silver and gold nanoparticles: effect of structure of carbohydrates and reducing agents on the size and morphology of the composites. Carbohydr Res 346: 651-658. [Crossref]

2. Shervani Z, Yamamoto Y (2011) Size and morphology controlled synthesis of gold nanoparticles in green solvent: Effect of reducing agents. Materials Letters 65: 92-95.

3. Shervani Z, Ikushima Y, Sato M, Kawanami H, Hakuta Y, et al. (2008) Morphology and size controlled synthesis of silver nanoparticles in aqueous surfactant polymer solutions. Colloid Polym Sci 286: 403-410.

4. Manabe K, Tanaka C, Moriyama Y, Tenjimbayashi M, Nakamura C, et al. (2016) Chitin Nanofibers Extracted from Crab Shells in Broadband Visible Antireflection Coatings with Controlling Layer-by-Layer Deposition and the Application for Durable Antifog Surfaces. ACS Appl Mater Interfaces 8: 31951-31958. [Crossref]

5. Li M, Wu Q, Song K, Cheng H N, Suzuki S, et al. (2016) Chitin Nanofibers as Reinforcing and Antimicrobial Agents in Carboxymethyl Cellulose Films: Influence of Partial Deacetylation. Sustainable Chem Eng 4: 4385-4395.

6. Muzzarelli RA (2010) Chitins and chitosans as immunoadjuvants and non-allergenic drug carriers. Mar Drugs 8: 292-312. [Crossref]

7. Ravi KMNV (2000) A review of chitin and chitosan applications. Reactive and Functional Polymers 46:1-27.

8. Sun W, Processing and microfabrication of self-assembled chitin nanofibers and composites. (2014) Master of Science thesis, University of Washington, Seattle.

9. Nair KG, Dufresne A (2003) Crab shell chitin whisker reinforced natural rubber nanocomposites. 1. Processing and swelling behaviour. Biomacromol 4: 657-665.

10. Oun AA, Rhim JW (2017) Preparation of multifunctional chitin nanowhiskers/ZnO-Ag NPs and their effect on the properties of carboxymethyl cellulose-based nanocomposite film. Carbohydr Polym 169: 467-479. [Crossref]

11. Lu Y, Weng L, Zhang L (2004) Morphology and properties of soy protein isolate thermoplastics reinforced with chitin whiskers, Biomacromol 5:1046-1051. [Crossref]

12. Kawano A, Yamamoto K, Kadokawa J (2017) Preparation of self-assembled chitin nanofiber-natural rubber composite sheets and porous materials. Biomolecules 7:47-57.

13. Jin J, Lee D, Im H, Han YC, Jeong EG, et al. (2016) Chitin nanofiber transparent paper for flexible green electronics. Adv Mater 28: 5169-5175.

14. Mushi NE (2014) Chitin nanofibers, network and composites-preparation, structure and mechanical properties. Doctoral thesis, KTH, Royal Institute of Technology, Stockholm.

15. Leertouwer HL, Wilts BD, Stavenga DG (2011) Refractive index and dispersion of butterfly chitin and bird keratin measured by polarizing interference microscopy. Optic Express 19: 24061-24066.

16. Takai S, Hoshimi N, Esaka T (2004) Synthesis of Tungsten,Molybdenum and Vamdium Bronzes by Mechanochemical Method Milling with Liquid Hydrocarbons. Electrochem 72: 876-879.

17. Macdonald JR (1987) Impedance spectroscopy JOHN WILEW \& SONS, New York.

Copyright: (C)2017 Shervani Z. This is an open-access article distributed under the terms of the Creative Commons Attribution License, which permits unrestricted use, distribution, and reproduction in any medium, provided the original author and source are credited. 